\title{
The Application of Ultrasonic Guided Wave in Grounding Grid Corrosion Diagnosis
}

\author{
Yiming Jiang, Wenguang Chen, Ying huang, and Hougui Chen
}

\begin{abstract}
Grounding grid corrosion diagnosis has always been a difficulty in the field of power system, which has aroused the concern of many scholars. In order to solve this problem, two-point method and partition method, which are based on ultrasonic guided wave theory and grounding grid structure features, are proposed in this paper. Two-point method excites guided waves at two points firstly. Secondly, to establish two equations based on the experiments. Then the location of the corrosion coordinates can be determined by the equations. The partition method is suitable for large and medium-sized grounding grid. In this method, the grid needs to be split into several blocks. Then Two-point method can be used in each block.
\end{abstract}

Index Terms-Corrosion diagnosis, grounding grid, ultrasonic guided wave.

\section{INTRODUCTION}

The grounding grid is an important facility to ensure the electrical equipment and personal safety. As the conductor buried in the ground, the grounding grid is easy to be corroded, resulting in a serious threat to the safety of operating personnel and electrical equipment [1]. There has been a lot of method for grounding grid corrosion diagnosis. The most important methods are based on the theory of electromagnetic field or the theory of circuit theory, and the later one is more widely used [2]-[3]. But in practice, subject to network structure and the limitations of the method itself, these methods cannot be used for accurate positioning. In recent years, the grounding grids corrosion diagnosis has always been a hot issue in the field of electrical engineering, but most of the study are nothing more than the above two methods.

From the last $1950 \mathrm{~s}$, there has been a non-destructive testing technology for Long-distance rapid detection. That is the ultrasonic guided wave technology, which is mostly used in the pipe inspection [4]. This paper discusses the application of ultrasonic guided waves in the detection of grounding rids.

\section{THE STRUCTURE OF GROUNDING GRID}

The propagation characteristic of ultrasonic guided wave is closely related to the physical characteristics and geometry

Manuscript received October 16, 2012; revised November 26, 2012.This work was supported in part by Wuhan University under Grant 111048638

The authors are with School of Electrical Engineering, Wuhan University 430072 China (e-mail:534928470@qq.com, chenwenguangc@163.com, $349566177 @$ qq.com, 83684787@qq.com). of the medium. Therefore, it's important to study the structure of grounding grid. According to the actual situation, the main materials used for grounding grid in our country are the flat steel and round steel [4]-[6] Fig. 1 (a) (b) is a schematic diagram of flat steel and round steel.

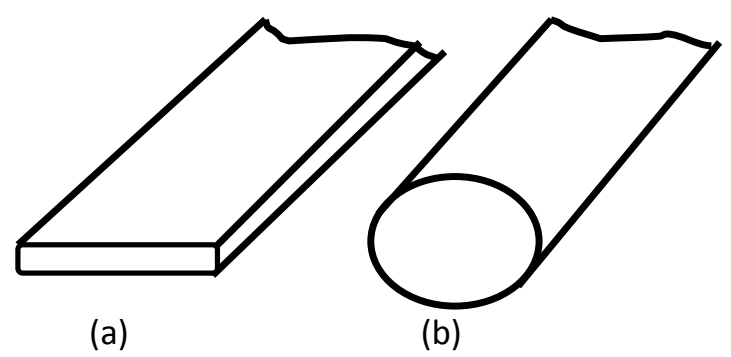

Fig. 1. Grounding grid material

China's electric power industry standard DL/T621-1997 ground connection of alternating current equipment make the following regulation for the smallest cross-sectional area of the material used for grounding grid [5].

TABLE I: THE Minimum Size of THE GROUNDING DEVICE

\begin{tabular}{lllll}
\hline Class & $\begin{array}{l}\text { Specificat } \\
\text { ion and } \\
\text { unit }\end{array}$ & Overground part & $\begin{array}{l}\text { Underground } \\
\text { part }\end{array}$ \\
\hline $\begin{array}{l}\text { Round } \\
\text { steel }\end{array}$ & $\begin{array}{l}\text { dia. } \\
\text { mm } \\
\text { Flat steel }\end{array}$ & 6 & 8 & $8 / 10$ \\
Anglion & $\begin{array}{l}\text { Orea mm } \\
\text { Thickness }\end{array}$ & 24 & 48 & 48 \\
mm steel & $\begin{array}{l}\text { Thickness } \\
\text { mm }\end{array}$ & 2 & 4 & 4 \\
Phickness & 2.5 & 2.5 & 4 \\
\hline
\end{tabular}

Remarks:

1) As for the diameter of the underground part of the round bar, the numerator and denominator corresponds to grounding device of overhead lines and power plants, substation.

2) As for the underground part of pipe wall thickness, the molecular denominator correspond to buried in the soil and buried in the indoor prime concrete floor

3) The section of overhead line tower grounding pole pinout should not be less than $50 \mathrm{~mm} 2$, and shall be hot dip galvanized.

The grounding grid is welded together by flat or round steel with low resistivity of solder. Fig. 2 is a schematic diagram of the grounding grid.

According to China's electric power industry standard DL/T621-1997 ground connection of alternating current equipment [5] and the IEEE Recommended Practice for the Grounding of Industrial and Commercial Power Systems[6], a well-designed grounding grid should be based on the 
ground resistance, potential of grounding connection, step potential difference and contact potential difference and other parameters, determine the specifications of the grounding grid and material parameters regulate by the standard. Then the specifications of the grounding grid and material parameters can be determined. The references [5]-[8] show that the side of grounding grid is often no more than $500 \mathrm{~m}$. For small grid, the side is only tens of meters. The whole grounding grid is buried in earth.

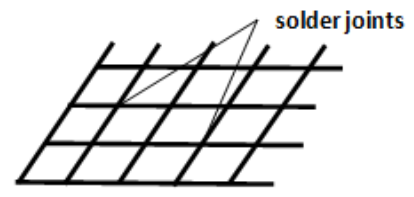

Fig. 2. Typical structure of grounding grid

\section{ANALysis OF Ultrasonic GUIDED WAVE ThEORY}

Fig. 3 shows a typical schematic diagram of the ultrasonic guided wave detection.

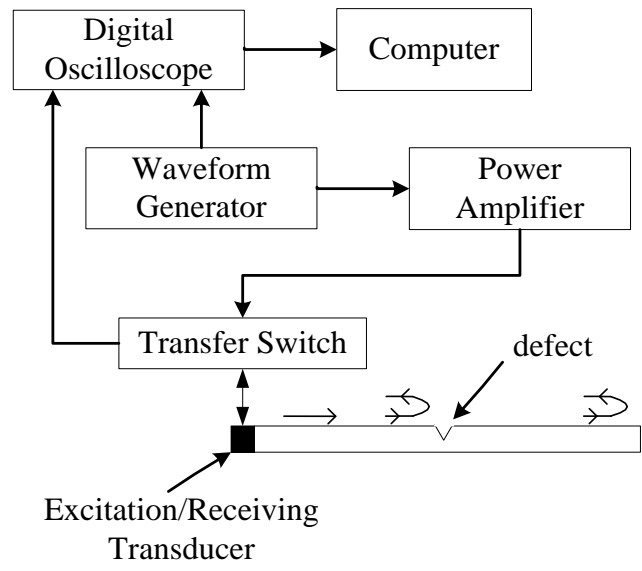

Fig. 3. Typical schematic diagram of ultrasonic guided wave inspection.

Just as Fig. 3 shows, ultrasonic is often excited and received at one point. When propagating in the medium (waveguide), the ultrasonic will form ultrasonic guided wave. The guided wave will echo at the point of defect. The location of the defect can be determined by the time difference of the reflection and incident wave [9].

Ultrasonic guided wave possesses the characteristics of long-distance propagation and low attenuation. Unlike traditional ultrasonic testing, ultrasonic guided wave just need excite signal at one point, then the information of the whole structure can be known. Therefore point-by-point detection is not necessary. However, guided wave also has the characteristics of multi-modal and dispersion, which leads to data analysis very complex. Therefore, to select a single-modal guided wave is quiet important [4]. Modal choice is established on the basis of the dispersion curves. As the grounding grid is welded together by flat or round steel, only the guided wave in the plate and bar is considered.

Lamb wave research the plane strain wave in the free version on which surface traction force is zero. The dispersion equation of Lamb's symmetric modal and antisymmetric modal is known as the Rayleigh-Lamb dispersion equation. The form of the equation is as follow:
[10]

$$
\begin{aligned}
& \frac{\tan (\beta h)}{\tan (\alpha h)}=-\frac{4 k^{2} \alpha \beta}{\left(\beta^{2}-k^{2}\right)^{2}} \\
& \frac{\tan (\beta h)}{\tan (\alpha h)}=-\frac{\left(\beta^{2}-k^{2}\right)^{2}}{4 k^{2} \alpha \beta}
\end{aligned}
$$

Equation (1) is suitable for symmetrical modal and equation (2) is suitable for antisymmetric modal. $\alpha$ and $\beta$ is given by:

$$
\alpha^{2}=\frac{\omega^{2}}{c_{L}}-k^{2} \quad \beta^{2}=\frac{\omega^{2}}{c_{T}}-k^{2}
$$

The analytical solution of Rayleigh-Lamb dispersion equation cannot be solved, but the numerical solution of the equation can be solved by referencing Ultrasonic Waves in Solid Media [10] authored by Joseph L.Rose.

Ultrasonic guided wave propagated in the bar shows the three models, whose names are longitudinal modal, reverse modal and bending modal [10]. Only longitudinal modal is studied in this paper.

Based on the knowledge of elasticity, sports formula for isotropic elastic medium (excluding physical strength) is as equation (4):

$$
\mu \nabla^{2} \bar{u}+(\lambda+\mu) \nabla(\nabla \cdot \bar{u})=\rho\left(\frac{\partial^{2} \bar{u}}{\partial t^{2}}\right)
$$

The vector field can be decomposed into a scalar potential function and a vector potential function by Helmholtz decomposition. Then equation (4) can be expressed as follows [4]:

$$
\begin{gathered}
\nabla^{2} \phi=\frac{1}{C_{L}^{2}} \frac{\partial^{2} \phi}{\partial t^{2}} \\
\nabla^{2} \psi=\frac{1}{C_{T}^{2}} \frac{\partial^{2} \psi}{\partial t^{2}} \\
C_{L}^{2}=\frac{\lambda+2 \mu}{\rho} \quad C_{T}^{2}=\frac{\mu}{\rho}
\end{gathered}
$$

The boundary condition of the problem is: on the surface of the bar

$$
\sigma_{r r}=\sigma_{r z}=0
$$

Equation (5) (6) can be ascribed to the solution of Bessel equation. A set of equations will finally be got by substitution of boundary condition. The matrix representation is as follows[10]:

$$
[C][A, B, C, D]^{T}=0
$$


By ordering the determinant zero, we can get the dispersion equation as equation (9) [10].

$$
\begin{aligned}
& \frac{2 \alpha}{a}\left(\beta^{2}+k^{2}\right) J_{1}(\alpha a) J_{1}(\beta a)- \\
& 4 k^{2} \alpha \beta J_{1}(\alpha a) J_{0}(\beta a)- \\
& \left(\beta^{2}-k^{2}\right)^{2} J_{0}(\alpha a) J_{1}(\beta a)=0
\end{aligned}
$$

$J_{0}$ and $J_{1}$ is Bessel function. $a$ represents the Radius of the bar. Dispersion curves can be calculated from equation (9) by substitution of the physical parameter of bar.

\section{GROUNDING GRID CORROSION DiAgNOSIS BASED ON ULTRASONIC GUIDED WAVE}

From the above analysis of the guided wave theory, we can see that board and bar ultrasonic guided wave theory can be applied to the corrosion diagnosis for flat and round bar material. The main obstacle when ultrasonic guided wave is applied to grounding grid corrosion diagnosis is that the grounding grid is a network, which leads to complex signal which is hard to analyze. Two methods are proposed as follows for this feature.

\section{A. Method One: Two-Point Method}

In order to simplify the problem, only uniform distributed grounding grid is considered. The two orthogonal steels at the edge of the grounding grid are taken for axis to establish a coordinate system. Fig. 4 is the schematic diagram.

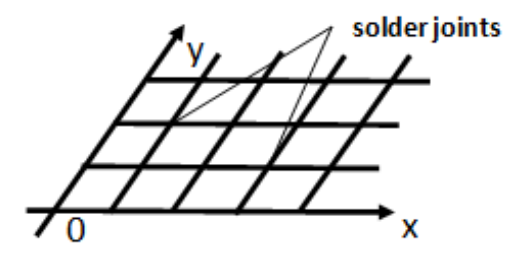

Fig. 4. Coordinate graph of grounding grid

After the establishment of coordinate system, the steels and solder joints should be numbered. The steels perpendicular to $\mathrm{x}$ axis are numbered $\mathrm{y}=0$ ( $\mathrm{x}$ axis), $\mathrm{y}=1$, $\mathrm{y}=2 \ldots$, and the steels perpendicular to $\mathrm{y}$ axis are numbered $\mathrm{x}=0(\mathrm{y}$ axis $), \mathrm{x}=1, \mathrm{x}=2 \ldots$...Solder joints are the coordinates where they are located.

At first, reflected wave of the node is ignored. Assuming that the corrosion point is $\left(x_{0}, y_{0}\right)$ and ultrasonic guided wave is excited at $(0,0)$. When the guided wave modes and frequencies are selected, the wave velocity can be known. Then the distance from the corrosion point to $(0,0)$ can be determined by the time of the reflection. If the distance is assumed to be $d_{1}$, then

$$
x_{0}+y_{0}=d_{1}
$$

Obvious, the coordinate of the corrosion cannot be determined only by equation (10). But if the experiment is done in other point again, such as $(1,0)$, , another equation can be get:

$$
\left|x_{0}-1\right|+y_{0}=d_{2}
$$

The coordinate of the corrosion can be determined when equation (10) is combined with equation (11). For the situation where more than one defect is existed, two-point method is also suitable for it. Actual operation can be detected a few points for the test results. But in practice, more than two experiments can be done to check the answer.

When the reflected wave of the node is considered, the signal is extraordinarily complex. However, if the grounding grid is uniform distributed, distance of any two adjacent nodes is the same. Therefore, every specific time the echo signals of solder joints will return. The signal is a regular interval signal in time domain.

\section{B. Method Two: Partition Method}

Two-point method is feasible in theory, but in practice, the energy of ultrasonic guided wave is limited. The energy may be reduced to zero before the wave spread to the end of the grounding grid. Therefore this method is not suitable for large and medium-sized grounding grid.

When the size of the grounding grid is too large, the grid can be split into several blocks. Fig. 5 shows a grounding grid split into 6 blocks. Two-point method can be used in each block.

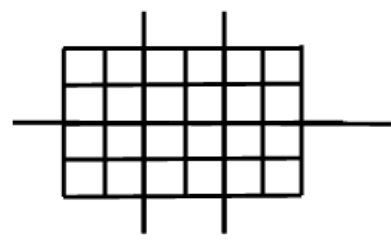

Fig. 5. The partition of grounding grid

\section{DISCUSSION}

Through decades of development, the ultrasonic guided wave technology has become an important non-destructive testing technology. However, this technology is only applied in some special occasion. For the traditional grounding grid corrosion diagnosis method, there are many problems that could not break through. Ultrasonic guided wave technology is a new method for the grounding grid corrosion diagnosis and there is little research on it.

The two methods proposed in this paper are just proved to be feasible in theory but there is no experimental study temporarily. The followings will be the issues what the research group focuses on.

1) The calculation of dispersion curves and the choice of ultrasonic guided wave modals in the grounding grid.

2) Ultrasonic guided wave detector designed for grounding grid.

3) Experiment on two-point method and partition method

\section{REFERENCES}

[1] C.-J. Cai, "Investigation and analysis on damage reasons of AC electric power grounding network," Electrical equipment, 2005, vol. 6, no. 4 , pp. $20-22$ 
[2] J. Liu, Grounding grid's failure diagnosis, Beijing : China Electric Power Press, 2011

[3] X.-H. Xiao and H. Liu, "Analysis of theory and method about the corrosionas well as the broken point of the grounding grid," Journal Of Chongqing University (Natural Science Edition), 2001, vol. 24, no. 3, pp. $72-75$.

[4] Z. Wang, Ultrasonic guided wave techniques and the application to ndt in pipes, Beijing University of Technology, 2002.

[5] The People's Republic of electric power industry, DL/T 621-1997 Ground connection of alternating current equipment.

[6] IEEE Std 142.-2007, IEEE recommended practice for grounding of industrial and commercial power systems.

[7] F. Qian, Design technique of grounding grid model for substation, North China Electric Power University, 2009

[8] F.-X. Tang, "Structure of ground net and measurement of grounding resistance," Sichuan Electric Power Technology, 2004, vol. 27, no. 3, pp. 33-34.

[9] C.-F. He, "Experimental study on defect detection in curved pipes using ultrasonic guided waves technique," China Mechanical Engineering, 2005, vol. 16, no. 18, pp. 1662-1665.

[10] J. L.Rose, Ultrasonic waves in solid media, cambridge: Cambridge University Press, 1999.

[11] Z.-Q. Liu, "Guided wave technology in ultrasonic non-destructive testing," Non-Destructive Testing, 1999, vol. 21, no. 8, pp. 367.

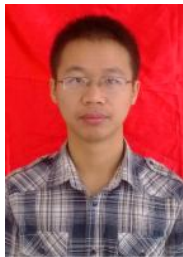

Yiming Jiang was born in June 1990. He is an undergraduate from Wuhan Univiesty. The project leade of "Experiment Study on the Method of Grounding Grid Corrosion Diagnosis Based on Ultrasonic Guided Wave ", which is one of Planning Projects of Innovative Experiment of National Undergraduate.

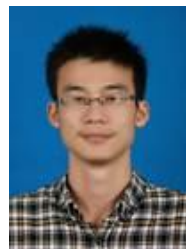

WenguangChen was born in December 1990. He is an undergraduate from Wuhan University.one of the member of " the study on the orientation ofgrounding grip corrosion based on ultrasonic guided wave "which is one ofThe National College Students' Innovative Training Project

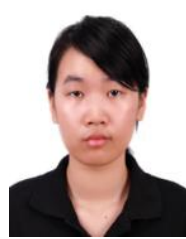

Ying Huang was born in December 1992. She is an undergraduate from Wuhan University, School of electric engineering, who is dedicated to the study of Automation of electric power systems field

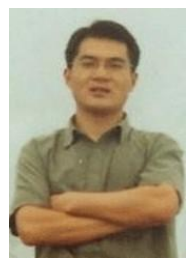

Hougui Chen was born in October 1973. He ia an associate professor of Wuhan University. The director of Hubei Society of Mechanical Engineering. He is working in non-destructive testing for years. 